\title{
La acreditación de la educación superior colombiana. Balance y perspectivas
}

\author{
ACCREDITATION OF COLOMBIAN HIGHER EDUCATION. REVIEW AND OUTLOOK \\ ACREDITAÇÃO DO ENSINO SUPERIOR DA COLÔMBIA. ANÁLISE E PERSPECTIVAS
}

Gloria Almeida Parra* / galmeidaparra@yahoo.com

Tulio Ramírez ${ }^{\star *}$ / tuliorc1@gmail.com

\section{Resumen}

Los procesos de autoevaluación para el registro calificado y la acreditación de alta calidad, se constituyen como herramientas de gran valor para determinar hasta qué punto una institución de educación superior cuenta con los elementos que le permiten estar al tanto de las necesidades que manifiesta la sociedad. En un momento donde es más fácil acceder a la información y a diferentes formas del conocimiento, es pertinente identificar la importancia de esos procesos, definiendo qué se ha logrado hasta el momento en Colombia y qué faltaría por lograr.

\section{Summary}

Self-evaluation processes with a view to obtaining qualified registration or accreditation of high quality constitute valuable tools to determine to what extent an institution of higher education has the elements that allow you to be aware of the needs says the company, at a time when it is easier to access information and different ways of acquiring knowledge. It is then appropriate to identify the importance of these processes, in addition to identifying what has been achieved so far in Colombia and which would fail to define or achieve in terms of educational processes.

\section{Resumo}

Auto-avaliação de processosqualificados para cadastramento e credenciamento de alta qualidade, constituemferramentas valiosas para determinar até que ponto umainstituição de ensino superior tem os elementos que lhepermitem estar ciente das necessidadesexpressas da sociedade, emum momento onde é mais fácil o acessoàsinformações e diferentes formas de conhecimento. É pertinente para identificar a importânciadestesprocessos, definindo o que foialcançado até agoranaColômbia e que aindatemos dealcançar.

\section{Palabras clave}

Educación superior, evaluación, docentes, acreditación, calidad

Keywords

Higher education, evaluation, teachers, accreditation, quality

\section{Palavras chave}

Ensino superior, avaliação, educação, acreditação, qualidade

\footnotetext{
* Especialista en Administración y Docencia Universitaria, candidata a Doctorado en Educación, Universidad Central de Venezuela. Coordinadora Académica Facultad de Comunicaciones, Artes y Diseño, Universidad de Santander, Bucaramanga.

** Sociólogo y Abogado. Universidad Central de Venezuela (Venezuela), Magister en Formación de Recursos Humanos (Universidad Católica Andrés Bello, Venezuela), Doctor en Filosofía y Ciencias de la Educación UNED-España (2006). Coordinador del Doctorado en Educación de la Universidad Central de Venezuela.
}

Fecha de recepción: 12 de mayo de 2012 / Fecha de aprobación: 8 de junio de 2012 


\section{Introducción}

Durante los últimos 20 años, luego de la promulgación de la Nueva Constitución Política de Colombia, en 1991, diversos sectores del acontecer nacional han contado con elementos y referentes claros que les han permitido transformarse y evolucionar. El sector educativo no ha sido la excepción y, si bien se han dado procesos que han tenido dificultades en su desarrollo y ejecución, hoy es posible identificar que existen unos lineamientos y políticas claras de evaluación de la educación en función de la búsqueda de la excelencia.

Diversos Decretos presidenciales, Resoluciones ministeriales y diversas herramientas jurídicas, han dado lugar al fortalecimiento de los procedimientos que deben adelantar las instituciones de educación superior (IES), partiendo de la base de la autoevaluación académica e institucional, como condición determinante para lograr el mejoramiento.

Estos procesos de autoevaluación se han fundamentado en el principio de autonomía universitaria, que le permite a las IES definir y establecer las condiciones internas de trabajo que les faculten para hacer frente a los requerimientos de las nuevas generaciones de profesionales o tecnólogos, quienes deben contar con elementos disciplinares que los constituyan en factores de transformación de la sociedad a partir del desarrollo de competencias, en donde cobra importancia la facultad de adquirir, transformar, adaptar y transferir conocimiento a partir de los recursos con los que se pueda disponer.

En este orden de ideas, es entonces importante mencionar que fenómenos como la globalización no son ajenos a la manera en que se deben desarrollar los procesos de enseñanza-aprendizaje, las actividades propias de la proyección social y los ejercicios relacionados con la investigación básica o la investigación aplicada. En esta vía, las tecnologías de información y comunicaciones cobran una gran trascendencia en la manera como se desarrollan los procesos educativos.

Las líneas que se presentan a continuación desarrollan los elementos a considerar al momento de llevar a cabo los ejercicios de evaluación de las IES en Colombia. Se argumentará por qué aspectos como el papel del docente, los lineamientos e indicadores de rendimiento que se desprenden de las propias IES y las herramientas que el medio ofrece para facilitar el ejercicio académi- co, son elementos que deben considerarse al momento de hacer ejercicios de autoevaluación, con el fin de determinar hasta qué punto una IES está preparada para afrontar los retos que plantea la hoy llamada sociedad del conocimiento.

\section{Fenómenos asociados a la evolución de la Educación en Colombia}

En los últimos años, la educación colombiana se ha transformado y se ha adaptado a pautas y referentes de evaluación que han sido planteados por diversas entidades reguladoras, bajo las directrices del Ministerio de Educación Nacional (MEN). Este ha sido un trabajo permanente y continuo, el cual, pese a los altibajos que precisaron ser corregidos, logró que las IES entraran en sintonía con los procesos de mejoramiento, ayudando a identificar mecanismos que permitan determinar si se está cumpliendo con el compromiso social en la formación de nuevas generaciones de profesionales.

Según Roa (2003), quien habla de la evolución de la educación superior en Colombia durante los últimos años, es pertinente mencionar la creación en 1968, del Instituto Colombiano para el Fomento de la Educación Superior (ICFES), encargado de promover la masificación de la educación, la vigilancia de las instituciones educativas y la elaboración de pruebas para medir el desempeño de los estudiantes; pruebas éstas que se han utilizado como requisito de ingreso a las IES.

Años más tarde, en 1980, se expide el Decreto Ley 80, a partir del cual se unifica el Sistema Nacional de la Educación Post-secundaria, se fijan los requisitos que debe tener un establecimiento destinado a la Educación Superior y se redefinen las funciones del ICFES frente a las universidades. El mismo autor ha señalado que durante esos veinte años, en América Latina se presentó, de manera paralela, un fuerte crecimiento de la cobertura y la apertura de un amplio número de IES de carácter privado.

En 1992 se promulgó la Ley 30, consecuencia de la Nueva Constitución Política de Colombia de 1991. Esta Ley permitió establecer la Autonomía Universitaria como factor determinante para la calidad educativa. La Ley, en su Artículo 3, manifiesta que se garantizará la autonomía universitaria, concepto del que se habla ampliamente en su capítulo 6, donde se la define como: 
[...] el derecho a darse y modificar sus estatutos, designar sus autoridades académicas y administrativas, crear, organizar y desarrollar sus programas académicos, definir y organizar sus labores formativas, académicas, docentes, científicas y culturales, otorgar los títulos correspondientes, seleccionar a sus profesores, admitir a sus alumnos y adoptar sus correspondientes regímenes y establecer, arbitrar y aplicar sus recursos para el cumplimiento de su misión social y de su función institucional (Congreso de Colombia, Ley 30).

En esa medida, la autonomía universitaria permite a las instituciones de educación superior generar estrategias para lograr su mejor posicionamiento en las regiones en donde desarrollan sus actividades, con lo que pueden definirse perfiles institucionales con los que los futuros profesionales puedan sentirse identificados.

A estos procesos se suma la creación del Consejo $\mathrm{Na}$ cional de Acreditación, conformado por académicos, antes que por funcionarios gubernamentales, y dedicado a definir los lineamientos a seguir por las IES en materia de autoevaluación, con miras a la obtención del Registro Calificado y la Acreditación.

Con relación a lo anterior, cabe anotar lo planteado por Gómez y Celis (2009), quienes exponen que el sistema de aseguramiento de calidad, que está coordinado por expertos en educación, ha permitido la identificación de unconjunto de condiciones académicas e institucionales (factores y características) que están concebidas para definir la educación de calidad. Estos procesos de diagnostico deben constituirse en un ejercicio voluntario y permanente de las instituciones educativas. De ahí que se han concebido diversos mecanismos, ajustados a las condiciones propias de cada institución, que permiten la medición de los parámetros que los evaluadores del sistema nacional de acreditación, consideran trascendentales para el logro de los objetivos en la educación.

Una vez la institución ha logrado alcanzar los niveles esperados de calidad, o al menos un punto cercano, es esperable que se constituya en una filosofía del "deber ser" de cada institución, la cual debe estar asociada a su misión, visión, valores, principios y, por supuesto, en concordancia con el Proyecto Educativo Institucional, aspecto que hace único un ente de educación.

\section{La Calidad de la Educación Superior:} Problemáticas y Estrategias

La evolución y transformación, posibles a partir de la Constitución Colombiana de 1991 y los planes decenales del Ministerio de Educación, han permitido que se pueda hablar y discutir, paulatinamente, en términos de una política de calidad que tiende al logro de la acreditación de programas o instituciones. Lo anterior ha hecho que las IES hayan generado los espacios para evaluar la forma como están llevándose a cabo sus procesos, así como establecer los medios que faciliten el acercamiento entre las instituciones educativas y las entidades encargadas de regular el cumplimiento de las condiciones mínimas para ofrecimiento de planes de estudio.

Esta nueva dinámica supone una comunicación más efectiva, comparada con lo que sucedía hace al menos diez años, cuando las instituciones educativas estaban simplemente a la espera de las disposiciones emanadas de las autoridades educativas nacionales. Así, en la actualidad, es posible consultar guías y lineamientos diseñados por las entidades reguladoras, fruto de la experiencia con las instituciones educativas colombianas en función de su medición y su evaluación, de manera que tanto dichas entidades, como las universidades y demás centros de educación superior, puedan trabajar en coherencia con las políticas gubernamentales.

Este acercamiento, inédito en la historia de Colombia, entre las IES y las instituciones de regulación de la educación, ha permitido a ambas instituciones mirarse internamente y determinar si lo ofrecido está a la altura de las necesidades del mundo actual, regulado por los procesos de globalización e internacionalización.

La política de calidad debe estar alimentada por los aportes que tanto la comunidad académica como la ciudadanía en general, puedan hacer, con el objeto de definir estrategias que sean pertinentes a la situación actual del país y que, en esa medida, permitan la sostenibilidad de las instituciones y su permanente búsqueda de la proyección, a partir de estándares que han sido determinados por entidades multinacionales que trabajan permanentemente en esta vía.

A pesar de todos los esfuerzos que se han realizado y de los logros que se han alcanzado, la definición de políticas públicas actualizadas de acuerdo con las 
cambiantes condiciones de los últimos años y la existencia de entidades rectoras de los procesos de registro calificado y acreditación, aún se perciben debilidades que deben ser trabajadas en proyectos de participación multisectorial, con el fin de incrementar los índices de productividad educativa y así mejorar los estándares de calidad de vida en Colombia.

Entre las principales fallas que deben ser corregidas están:

la baja cobertura de la educación (no sólo la educación superior, sino también, y con mayor preocupación, la educación básica y media); la poca existencia de facilidades para el acceso a créditos educativos; el futuro de la universidad pública de continuarse con los modelos de gestión y financiación basados sólo en los aportes del Estado para su sostenimiento; la falta de integración de la educación media con la educación superior y de éste con el mercado laboral, así como la poca inversión gubernamental en educación superior e investigación (Gómez y Celis, 2009, p. 88).

Cabe recordar que el Decreto 2566 del 2003, expedido por el MEN de Colombia, planteó los parámetros para evaluar la calidad en la educación superior. Hoy contamos con el Registro Calificado, que es de carácter obligatorio para todos los programas académicos, y la Acreditación, que es de carácter voluntario y puede ser aplicable tanto a programas académicos específicos como a instituciones educativas. En ambos casos se establecen estándares que permiten verificar las condiciones de calidad que se deben ofrecer para satisfacer las expectativas y necesidades de lasociedad.

Por otra parte, el ICFES cuenta con la responsabilidad de diseñar y establecer las pautas de evaluación de los procesos de formación en educación superior, mediante el uso de pruebas evaluativas que miden el conocimiento de los estudiantes de últimos semestres en los niveles tecnológico y profesional (ICFES, 2011). Estas pruebas, establecidas por el Decreto 3963 del 2009, inicialmente se denominaron Exámenes de Calidad de la Educación Superior, ECAES, hoy reciben el nombre de Pruebas Saber Pro. En su implementación han tenido un proceso de perfeccionamiento luego de una serie de altibajos, entre los que se destaca el hecho de la falta de claridad frente a la obligatoriedad de su presentación o la utilización de los resultados como factor determinante para las convocatorias laborales o de becas de posgrado a las que aspiraban los recién egresados.

Roa (2003), plantea que la aparición y evolución de estos exámenes no ha estado exenta de discusiones académicas, dado que los resultados obtenidos por los estudiantes de cada IES están siendo presentados como referentes de la calidad educativa, sin tener en cuenta variables y factores que están asociados con las condiciones y características en las que se desarrollan los procesos educativos en las instituciones (p. 31). Además, se cuestiona el hecho de que no miden las competencias profesionales que deben ser desarrolladas por los egresados en cualquier programa académico.

Otra crítica importante tiene que ver con el hecho de que este tipo de pruebas, tal y como están concebidas, podría llegar a limitar la posibilidad de las IES para desarrollar sus proyectos educativos institucionales de forma autónoma, tendiendo hacia la homogenización de planes curriculares acordes con las temáticas evaluadas en el examen, con el fin de tener resultados que sean catalogados como buenos o favorables, descuidando diversos proyectos y procesos alternos y complementarios que en todas las IES se desarrollan y que forman parte de su impronta institucional.

Otro aspecto que ha generado una interesante discusión es el propio concepto de calidad académica. Este es un tema de discusión permanente entre académicos y entidades reguladoras, dado que aún existen diferencias frente a su conceptualización, por la misma complejidad del tema y el diverso y cada vez más amplio número de variables que afectan los procesos educativos y las condiciones de desarrollo de las IES, así como los programas académicos de orden tecnológico o profesional.

Roa afirma al respecto que en cuanto al tema específico de la acreditación, ésta sigue respondiendo a la necesidad de fortalecer la calidad de la Educación Superior. Las IES al momento de alcanzar altos niveles de calidad, obtienen ventajas competitivas frente a otras instituciones educativas. Esto, además de dar respuesta a los parámetros que se plantean en el ámbito internacional, mejora las condiciones educativas de la población en general. 


\section{La acreditación de las IES en Colombia. Consideraciones críticas}

Son muchos y muy variados los aspectos asociados al tema de la evaluación institucional y al programa de acreditación, tantos, que van más allá del simple análisis interno de las condiciones en que se opera en las IES. Sin embargo, el camino recorrido hasta la fecha ha permitido definir parámetros o lineamientos que las instituciones educativas han adoptado y adaptado para establecer qué tan cercanas están al concepto de calidad manejado por los entes reguladores, pero es inevitable hablar de la identificación de algunas falencias que aún no han sido resueltas y que reclaman una solución que permita que estos procesos conduzcan a una calidad redundante y acorde con lo que la comunidad, y el medio en general, necesita.

Veamos algunas críticas que Gómez y Celis (2009) formulan frente al sistema que orienta el proceso de evaluación, a partir de los resultados obtenidos en un estudio diagnóstico sobre el sistema de evaluación que conlleva a la acreditación, realizado en el año 2007 por el Centro de Investigaciones para el Desarrollo de la Universidad Nacional de Colombia:

a. El sistema procedimental del Consejo Nacional de Acreditación (CNA), basado en la evaluación y verificación de ocho grandes "factores" de calidad, por el sistema de pares externos, es de baja eficacia - y de alto costo - en relación con el volumen total de programas existentes en la educación superior. Al ritmo actual de funcionamiento de este sistema, serán necesarias varias décadas y altísimos costos para acreditar un porcentaje significativo de los programas actuales, además de los programas cuya acreditación inicial haya caducado y requiera reacreditación. Es evidente, entonces, que este sistema es inoperante, ineficiente y de escaso costo/ beneficio.

b. En el origen del CNA se incurrió en el grave error de definir cada programa curricular como la unidad básica de acreditación, legitimando de esta manera el proceso que se estaba dando de proliferación de programas (los que se duplicaron en los primeros años de vigencia de la Ley 30), e ignorando los intereses que impulsaban dicha proliferación, tales como: estrategias de mercadeo de programas, competencia entre instituciones por matrículas, y la subdivisión arbitraria de áreas del conocimiento con el propósito de ofrecer nuevas denominaciones de programas.

c. Hay problemas metodológicos que cuestionan la validez y confiabilidad de las evaluaciones de los programas, asociadas a la selección de pares evaluadores y sus diversos criterios de evaluación. Otro de los problemas identificados tiene que ver con el rol de par y el proceso de capacitación que se recibe para asumir esta responsabilidad. No es suficiente ser profesor en el nivel superior para desempeñar con competencia y objetividad el rol de par evaluador, dado que deben determinarse si los pares cuentan con las competencias que les permitan lograr el análisis, la ponderación y la evaluación de una gran cantidad de aspectos que están asociadas a los procesos evaluativos de un programa académico o de una institución educativa en función de la acreditación (Gómez y Celis, 2009, p. 4).

La selección de un par académico tiene en sí misma una gran relevancia, pues este no sólo debe tener competencias disciplinares, sino también ser reconocido en el medio o gremio en el cual se desempeña, llegándose a plantear que debe ser, como un paradigma, digno de imitar. Al respecto Gómez y Celis (2009) afirman que:

La evaluación y ponderación de un programa de formación, según los ocho factores de calidad, es una tarea $-y$ responsabilidad - que solo puede ser adecuada y objetivamente realizada por académicos de amplio reconocimiento y trayectoria en un área del saber, sobre todo, con extensa experiencia en dicha tarea de evaluación. La competencia evaluativa no se improvisa, es el resultado de la acumulación de experiencia cualificada y reflexiva sobre el complejo asunto de la evaluación. Por esta razón, en la experiencia internacional, las funciones de evaluación y acreditación de programas e instituciones son realizadas por entidades especializadas en estas funciones, y no por pares que no han sido seleccionados según criterios de idoneidad y experiencia en la función de evaluación sino por estar presentes en una base de hojas de vida (p. 92). 
Los procesos de evaluación no pueden tomarse de manera superficial, dado que de las características de la metodología empleada y de la perspectiva que tengan los pares académicos que revisen las condiciones de operación, de una institución educativa o de un programa académico, se determinará su pertinencia social, económica y académica, así como la valoración del impacto que en términos del desarrollo regional se podría generar por parte de los egresados de un programa académico u otro.

Otro de los aspectos críticos más señalados, tiene que ver con el hecho de que las guías que el CNA elabora para el desarrollo de procesos de autoevaluación y de mejoramiento institucional, no exponen elementos puntuales que permitan hacer una evaluación bajo los mismos parámetros para todas las instituciones, sino que se resalta el desarrollo de evaluaciones de tipo cualitativo antes que cuantitativo, aspecto éste que puede dar lugar a una mayor subjetividad, por parte, tanto de los profesionales que al interior de las instituciones educativas están a cargo de hacer el seguimiento de los procesos de evaluación, como de los pares que evalúan, con perspectivas diferentes, los procesos académicos y en general de orden institucional que se desarrollan en una IES.

\section{Algunos de los retos que deben enfrentar las IES en Colombia}

A lo largo de este documento se han planteado diversos elementos asociados a los procesos de autoevaluación institucional, con miras a lograr el reconocimiento que, para el caso de Colombia, radica en la obtención del Registro Calificado y, en los casos en que las instituciones así lo decidan, la solicitud de la acreditación de calidad.

Sin embargo, es conveniente exponer que estos ejercicios, si bien se desarrollan con base en los lineamientos que el Ministerio de Educación y entidades reguladoras, como el Consejo Nacional de Acreditación, plantean, no son de relevancia únicamente local, sino que responden a la necesidad de satisfacer las necesidades de mejoramiento que le permitan a Colombia evolucionar permanentemente, a fin de ser clasificado a escala internacional como un país con alta calidad educativa.

Este interés por lograr un reconocimiento, a partir de la comparación basada en parámetros de medición, no sólo en el ámbito de la educación sino en situaciones que tienen que ver también con lo productivo, lo cultural o lo social, está dado por el impacto que la globalización causa en las actividades y en los procesos que se adelantan en diferentes escenarios; por el hecho de reconocer que ya no sólo dependemos de lo que sucede en el entorno inmediato, sino que se reciben influencias de contextos en apariencia distantes desde lo geográfico, pero cercanos en los objetivos que persiguen. En referencia a la educación, García (1996) manifiesta que:

En los nuevos contextos en los que la educación se está viendo imbricada, dos de los aspectos que mayor relevancia y velocidad están tomando son los procesos de integración y globalización dentro de lo que significa el nuevo valor del conocimiento. Por su parte, la globalización del conocimiento y la integración educativa no son excluyentes, ya que en muchos casos, para optimizar los beneficios que pueden derivar de los procesos de globalización se hace necesario el haberse organizado integradamente a menor escala (p. 11).

Ahora bien, siguiendo el planteamiento de García, las IES deben velar porque, a la par de los ejercicios de autoevaluación que deben adelantar con miras a lograr el registro calificado o la acreditación (o ambas), sus planes de mejoramiento deben incluir elementos de desarrollo institucional que les permitan estar a tono con los movimientos educativos que se gestan en otras latitudes, las formas de interacción que se están dando entre instituciones educativas y sus estudiantes, así como la manera en que los docentes están asumiendo su papel de orientadores y no de simples transmisores de información.

Ante ese reto, los países latinoamericanos en general y Colombia en particular, requieren identificar en qué estado actual se encuentran en materia de educación y, sobre esa base, plantear cómo y cuándo lograr las metas que se tracen, con el fin de satisfacer las expectativas que en materia educativa marcan la pauta las grandes instituciones educativas en el mundo.

Sin embargo, no se trata de copiar modelos que hayan funcionado en otros países. Se debe construir un modelo específico a partir de un ejercicio donde se inte- 


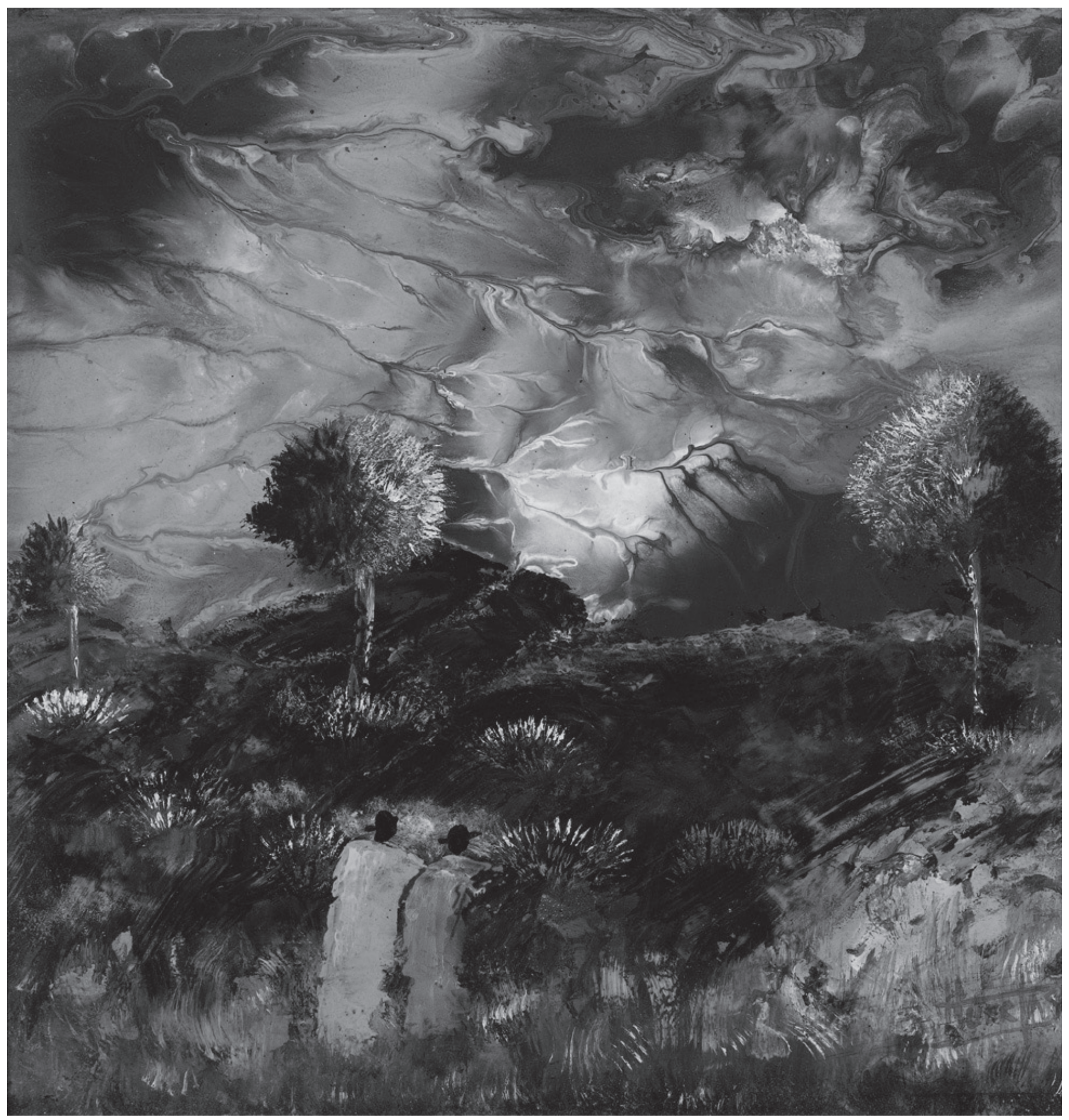

Segundo Arístides Huertas Torres ॥ Título: Paisaje Andino ॥Técnica: óleo sobre tela » Dimensiones: $100 \mathrm{~cm} \times 100 \mathrm{~cm}$ 
gren no sólo las autoridades en materia de educación y las IES, sino también los miembros de la sociedad que requieren de una participación más activa de las instituciones de educación superior, en función de la resolución de problemáticas de la cotidianidad. Aquí el reto consistiría en pensar global, pero actuar local.

En concordancia con esto, es necesario hablar de una de las exigencias que debe cumplir la educación superior: la pertinencia. Esta exigencia debe desarrollarse de la mano con la calidad académica, dado que las dos son ineludibles en una educación superior que busque responder a las necesidades del mundo de hoy. Además del planteamiento generalizado que la universidad debe atender a las necesidades del sector productivo, también debe actuar en función del desarrollo cultural, responder a los desafíos que la sociedad en general le plantea y, en esa medida, establecer claramente el papel que la universidad tiene en la sociedad y lo que la sociedad espera de ella. En este sentido, Tünnerman (2008) manifiesta que:

El concepto de pertinencia de la Educación Superior ha evolucionado hacia una concepción amplia de la misma y a su estrecha vinculación con la calidad, la equidad, la responsabilidad social, la diversidad, el diálogo intercultural y los contextos en que se desenvuelve.

Pertinencia y calidad deben marchar siempre de la mano, pues la pertinencia no se logra con respuestas educativas mediocres o de baja calidad. A su vez, los esfuerzos encaminados a mejorar la calidad de la Educación Superior no pueden omitir la valoración de su pertinencia. En los procesos de evaluación institucional, la valoración de la calidad y de la pertinencia social, deberían recibir la misma atención (p. 2).

Teniendo en cuenta lo anterior, es importante entonces establecer que los procesos de autoevaluación que adelantan las IES, con miras a lograr su registro calificado o la acreditación de alta calidad, deben estar diseñados y desarrollados en función de medir hasta qué punto se está cumpliendo con la función social que deben desempeñar y, en consecuencia, establecer si tecnológicamente están capacitados o preparados para afrontar los retos que supone el mantener un nivel de calidad de permanente mejoramiento.
El papel de la investigación y las Tecnologías de la Información y las Comunicaciones

Los avances en las Tecnologías de la Información y las Comunicaciones (TIC's), han sido otro factor que ha incidido en la calidad y la cobertura de la educación superior. Para una IES que imparte su enseñanza de forma "tradicional", el cambio a una que desarrolle parte o la totalidad de sus procesos mediante el uso de las nuevas tecnologías, requiere de transformaciones que van más allá de la adquisición de equipos y sistemas de comunicación; de acuerdo con lo planteado por Hallak y Poisson (2007):

[...] pasar a usar nuevas tecnologías requiere una importante revisión de las actividades y una ingente revisión en infraestructuras totalmente nueva para desarrollar y ofrecer nuevos cursos. Las principales decisiones necesarias para emprender estos cambios no pueden adoptarse ni a la ligera ni rápidamente (p. 111).

La experiencia ha demostrado que las instituciones que no se han preparado suficientemente para enfrentar los cambios que implica la introducción de las tecnologías de información y comunicaciones, que están vigentes y son de uso masivo en contextos diferentes al académico, ven afectados sus estándares de calidad, dado que el compromiso no puede reducirse sólo al componente académico, sino que debe afianzarse en una estructura administrativa sólida, dispuesta a hacer inversiones con una expectativa de retorno en el mediano y largo plazo, tiempo de espera que en muchos casos no se tiene previsto, dado que se generan expectativas muy amplias que se espera lograr en el corto plazo.

Siguiendo los planteamientos de Hallak y Poison (2007), es inevitable afirmar que: "si las universidades de educación a distancia no afrontan de un modo positivo el reto de fusionar y desarrollar continuamente las tecnologías, pueden perder estudiantes y el apoyo gubernamental" (p. 111). Además, las tendencias en educación han permitido identificar que un segmento cada vez mayor de la población solicita el acceso a la educación. Esto implicará, además de generar condiciones apropiadas para que los aspirantes a desarrollar un curso de nivel tecnológico o profesional puedan acceder a la educación, que las instituciones aumen- 
ten en tamaño, lo que incrementa la complejidad en su administración y, por ende, una mayor presión para generar procesos de calidad.

Por tanto, en la medida en que una institución crezca tanto en número de estudiantes como en infraestructura y recursos, mayor será su responsabilidad con el medio, que debe traducirse en procesos de Proyección Social (denominados también de Extensión) donde se logre un mayor impacto social, gracias a las características de los proyectos asociados a educación continua, servicio a la comunidad, prácticas formativas o investigación, con fines a mejorar las condiciones de desempeño de un núcleo poblacional determinado.

Así mismo, la investigación debe desarrollarse de una forma más compleja, estructurada y organizada, donde no sólo baste con que los estudiantes realicen un buen trabajo de finalización, sino que las directivas académicas fomenten espacios y condiciones para que los docentes universitarios establezcan grupos de investigación que, para el caso de Colombia, sean reconocidos por COLCIENCIAS como entidades válidas para el desarrollo de proyectos de generación, transformación, adaptación y/o aplicación del conocimiento, con lo que se adelantarían actividades denominadas como investigación en sentido estricto.

\section{Conclusiones}

Ginés, refiriéndose al reto que tienen las instituciones de educación superior en cuanto a ponerse a tono con las nuevas exigencias del entorno nacional e internacional, expresa lo siguiente: "la sociedad está sufriendo mutaciones muy profundas, y será necesario que la universidad se adapte a ellas si no quiere verse convertida en una institución obsoleta que no responda a las demandas sociales" (Ginés, 2004, p.14). De ahí que las instituciones de educación superior no pueden ser ajenas a los cambios y las transformaciones que se dan en el mundo, por ello no pueden manejar los mismos esquemas de trabajo que se dieron en el momento histórico en que fueron creadas.

Las transformaciones que ha logrado el proceso de regulación y medición de las instituciones de educación superior en Colombia, han permitido que las IES identifiquen la forma como pueden realizar su au- toevaluación, aplicando el principio de la autonomía universitaria. Esto, ha significado que las instituciones educativas definan planes de mejoramiento que les permitan el logro de niveles de calidad aceptados por las autoridades académicas del caso.

Las instituciones de educación superior y las entidades reguladoras de los procesos que allí se desarrollan, cuentan hoy con formas de comunicación más efectivas, que permiten el conocimiento de lineamientos y documentos guías para orientar los procesos de evaluación que se desarrollan en las instituciones, teniendo claridad frente a los parámetros que son exigidos. Esto ha contribuido a que se pueda establecer un lenguaje común y, en esa medida, hacer más eficiente el ejercicio evaluativo realizado por pares externos.

Las políticas de calidad deben ser definidas de acuerdo con lo que la ciudadanía requiere de una institución de educación superior, así como de los aportes que al respecto pueden generar los miembros de la comunidad académica. Esto, conlleva a lograr que los procesos institucionales puedan lograr la proyección y la pertinencia social con la que deben ejecutarse las actividades propias del quehacer académico.

Si bien se han logrado avances en la forma de evaluar la actividad académica en las universidades y demás centros de educación superior, se requiere aunar esfuerzos para que haya una participación más amplia por parte de actores diferentes de la sociedad, con el fin de lograr soluciones que tiendan hacia lo holístico y no se limiten a brindar elementos que tengan aplicabilidad en el corto o mediano plazo.

A la par de los procesos de evaluación institucional que se deben adelantar para lograr la acreditación, no son menos importantes los procesos de evaluación de los estudiantes que se encuentran en el último año de formación tecnológica o profesional, dado que los resultados obtenidos en el presente del Examen Saber Pro (antes ECAES), constituyen un referente para determinar en qué forma y hasta qué punto la institución educativa ha cumplido con su función de transmisión del conocimiento. Los resultados de estas pruebas, además, permiten determinar si el plan de estudios es pertinente con los requerimientos actuales o si se hace necesario adelantar procesos de actualización docente. 
No cabe duda de que la educación actual debe responder a necesidades de tipo global. Ya no basta con conocer sobre las condiciones del contexto inmediato, puesto que las exigencias a escala profesional trascienden el plano de las distancias de tipo geográfico, debido a que los actuales profesionales o tecnólogos deben desarrollar habilidades que les permitan hacer uso de la información que tienen, transformarla y adelantar acciones que les permitan el logro y apropiación de nuevos conocimientos que suplan necesidades del ambiente en donde se desarrollen sus actividades profesionales.

\section{Referencias}

Asamblea Nacional Constituyente. (1991, Julio). Constitución Política de Colombia. Gaceta Constitucional, 116. Obtenido en Abril de 2011, desde, http://www.secretariasenado.gov.co/ senado/basedoc/cp/constitucion_politica_19w91.html

Congreso de Colombia. (1992, Diciembre). Ley 30. Por la cual se organiza el servicio público de la Educación Superior. Diario Oficial, 40.700. Obtenido en Abril de 2011, desde, http://www.secretariasenado.gov.co/senado/basedoc/ley/1992/ley_0030_1992. html

García, C. (1996). Conocimiento, educación superior y sociedad en América Latina. Caracas: Nueva Sociedad. Obtenido en Mayo de 2011, desde, http://carmengg.dered.com/images/stories/ documentos/conocimiento/Cap\%201\%20Globalización\%20 e\%20lntegración.pdf

Ginés, J. (2004). La necesidad del cambio educativo para la sociedad del conocimiento. Revista Iberoamericana de Educación, 35, 13-37. Obtenido el 10 de Abril de 2011, desde, http://www. rieoei.org/rie35a01.htm

Gómez, V., y Celis, J. (2009). (Julio-Diciembre). Sistema de aseguramiento de la calidad de la educación superior: consideraciones sobre la acreditación en Colombia. Revista Colombiana de Sociología, V. 32, No 2. Bogotá: Universidad Nacional de Colombia. Bogotá. Extraído desde, http://www.humanas.unal.edu.co/img/ Nuevo/revista_colombiana_sociolog\%C3\%ADa/32/04-(GomezCelis_Sistema-de-aseguramiento).pdf

Hallak, J., y Poisson, M. (2007). Fraude académico, acreditación y garantía de la calidad: lecciones aprendidas del pasado y retos del futuro. La educación superior en el mundo. Bogotá: Mundiprensa.

Instituto Colombiano para la Evaluación de la Educación (ICFES). (2011). Definición y objetivos - SABER PRO (antes ECAES). Oferta de exámenes para 2011-1. Obtenido en Abril de 2011, desde, http://www.icfes.gov.co/index.php?option=com_content\&task= view\&id=351\&ltemid $=445$
Las tecnologías de información y comunicaciones constituyen hoy por hoy una herramienta de gran importancia al momento de mejorar la forma como se desarrolla la educación en las IES, porque permiten trascender hacia situaciones en las que no hay limitaciones de espacio o tiempo, donde las formas de captar el conocimiento se hacen de forma más inmediata y en las que se logra la interacción simultánea con actores que no necesariamente formen parte del contexto académico, pero que pueden hacer aportes de importancia en el ejercicio de enseñanza-aprendizaje.
Ministerio de Educación Nacional. (1980). Decreto 80. Por el cual se organiza el sistema de educación postsecundaria. Obtenido el 1 de Abril de 2011, desde, http://www.mineducacion.gov. co/1621/articles-102556_archivo_pdf.pdf

Ministerio de Educación Nacional. (2003). Decreto 2566. Por el cual se establecen las condiciones mínimas de calidad y demás requisitos para el ofrecimiento y desarrollo de programas académicos de educación superior y se dictan otras disposiciones. Obtenido en Abril de 2011, desde, http://www.mineducacion. gov.co/1621/articles-104846_archivo_pdf.pdf

Ministerio de Educación Nacional. (2009). Decreto 3963. Por el cual se reglamenta el Examen de Estado de Calidad de la Educación Superior. Obtenido en Abril de 2011, desde, http://www. mineducacion.gov.co/1621/articles-205955_archivo_pdf_decreto3963.pdf

Roa, A. (2003). Acreditación y evaluación de la calidad en la educación superior colombiana. Estudio realizado para IESALCUNESCO. Obtenido el 7 de marzo de 2011, desde, http://www. cedus.cl/files/acr_co_roa.pdf.

Tünnerman, C. (2008). Nuevas perspectivas de la pertinencia y calidad de la educación superior. Boletín IESALC Informa de Educación Superior, 207. Obtenido el 15 de mayo de 2011, desde, http://www.iesalc.unesco.org.ve/index.php?option=com_content $\&$ view=article \&id=2029\%3Anuevas-perspectivas-de-la-pertinencia-y-calidad-de-la-educacion-superior \&catid=126\%3Anoticiaspagina-nueva\&ltemid $=712 \&$ lang $=$ es

Velásquez, F., y Ospina, L.A. (2009). Efectos del modelo de aprendizaje experiencial y sus pedagogías asociadas en el logro de las metas de aprendizaje por parte de los estudiantes del curso de liderazgo de pregrado en ICESI. Cali: Universidad ICESI. 


\section{Diálogo del conocimiento}

Los doctores Gloria Almeida Parra y Tulio Ramírez hacen un muy interesante recorrido de los últimos 20 años sobre los procesos de acreditación en las Instituciones de Educación Superior, lo que resulta revelador y abre el debate hacia nuevas posturas, sobre todo en la coyuntura de una eventual reforma de la Ley 30 de la Educación Superior de 1992, en los años que vienen y que el Gobierno Nacional se vio obligado a retirar en el inmediatamente anterior, sobre todo porque lesionaba profundamente la autonomía universitaria, disfrazaba lo que es un derecho constitucional para convertirlo en un servicio financiando ya no por el Estado sino por fondos privados, públicos y mixtos al mejor modelo norteamericano. El artículo hace una revisión de los proceso de acreditación, libres, a los que pueden acceder los programas, pero siguiendo las condiciones de la política de la calidad propuestas y diseñadas por el Consejo Nacional de Acreditación, CNA, bajo la directriz del Ministerio de Educación Nacional, MEN, y que se mueven entre el eje de la autoevaluación y la acreditación, que permiten a las instituciones demostrarse a sí mismo, ante la sociedad y ante los entes reguladores del Estado que reúne las condiciones para seguir funcionando como Institución de Educación Superior. Sin embargo, habría que cuestionar de qué forma dicha homogeneidad de procesos son adversos a la Universidad Pública toda vez que esta depende de los recursos del Estado para la contratación de maestros que cada vez son menos en términos de docentes de planta, y a recibir estudiantes en mayor cantidad bajo las políticas de cobertura en condiciones de hacinamiento y pobreza institucional, lo cual afecta directamente la política de calidad, si de investigación, innovación pedagógica, calidad en los procesos pedagógicos y tecnológicos se trata. El artículo cita a Gómez y Celis, 2009, quienes sostienen que algunas de las fallas que deben corregirse son la "baja cobertura", "la poca existencia de facilidades para el acceso a créditos educativos" y el "el futuro de la universidad pública de continuarse con los modelos de gestión y financiación basados sólo en los aportes del Estado para su sostenimiento", lo cual parecería legitimar, antes que cuestionar profundamente la política pública de educación, la necesidad de un modelo privatizador, cuando lo que se requieren son más universidades públicas, gratuidad en la educación reclamado como derecho, y un modelo de universidad que responda a la construcción de país interpretando sus necesidades y no la complacencia a políticas externas. La pregunta que deberíamos hoy plantearnos es ¿qué es lo público de la universidad pública? Sin embargo, el artículo es crítico al cuestionar la estandarización de las pruebas de Estado, Saber Pro, que afectan la autonomía de los proyectos institucionales; así como la ineficacia de entes reguladores como el CNA, la proliferación de programas en las Instituciones de Educación Superior sin otra motivación que la competencia con otras instituciones; la atomización del conocimiento; los criterios de selección de los pares académicos, así como sus procesos metodológicos. Del mismo modo señala problemáticas muy importantes relacionados con los procesos de internacionalización y globalización que hacen, como se dijo anteriormente, abrir nuevos debates.

Edilson Silva Liévano 